ISBN 978-981-11-0008-6

Proceedings of 2016 6th International Workshop on Computer Science and Engineering

(WCSE 2016)

Tokyo, Japan, 17-19 June, 2016, pp. 559-562

doi: $10.18178 /$ wcse. 2016.06 .094

\title{
Portable ECG Real Time Signal Monitoring Device
}

\author{
Boonchana Purahong ${ }^{1+}$, Somsin Tongkrairat ${ }^{1}$, Hideyuki Nomura ${ }^{2}$, Chisato Kanamori ${ }^{3}$, Hisayuki \\ Aoyama ${ }^{3}$ and Vanvisa Chutchavong ${ }^{1}$ \\ ${ }^{1}$ Faculty of Engineering, King Mongkut's Institute of Technology Ladkrabang, Bangkok, Thailand \\ ${ }^{2}$ Department of Communication Engineering and Informatics, University of Electro-Communications, \\ Tokyo, Japan \\ ${ }^{3}$ Department of Mechanical Engineering and Intelligent Systems, Graduate School of Informatics and \\ Engineering, University of Electro-Communications, Tokyo, Japan
}

\begin{abstract}
This paper introduces about the application of Embedded system for analyzing heart rate signal in ECG (Electrocardiography) format using Microcontroller. Microcontroller must process signal in DSP method in this case the heart rate signal must be processed in real-time. Our system can analyze heart rate to find the frequency of heart beat and show ECG signal and heart rate on display of Microcontroller board. To obtaining heart rate signal we use electrode placed on body in lead 2 orientations. We decide to make our system portable and can run on battery all over a day for recording signal to analyze during a day.
\end{abstract}

Keywords: ECG sensor, embedded system, DSP.

\section{Introduction}

This article is describing our ECG Real Time Signal monitor and analyse system [1]. It can analyse the heart rate signal in ECG (Electrocardiography) format real-time and also display ECG signal waveform in graph format. We analyze heart rate signal for checking heart signal that are there some problem with heart and calculate heart beat rate [2]. We develop that system in small size for mobility by $10 * 15 \mathrm{~cm}$-square size. With $2000 \mathrm{mAh}$ battery the system can operate over a day continuously. Thus, it is suitable for monitor heart signals of a heart disease patient along 24 hours [3].

From statistic in 2014 Thai people die from heart disease more than 50000 or 6 people per hour. If there is no act it will increase from 17 to 23 million people die from heart disease in 2030 [4]. Therefore, precisely analysis of heart signal is necessary for heart disease patient. And another application of this system is to record heart signal and analyzed data to the patient's doctor. Doctor can monitor patient heart signal to predict and notices symptom that are happened.

\section{Heart Rate Measurement}

\subsection{ECG signal model}

There is various heart signal measurement method in nowadays. ECG is the one of common use methods to measure heart signal. ECG signal model is an electrical signal from the heart muscle. There is electrical signal sending to the heart muscle to signal heart for beating. Hence, the signal is a representative of heart working signal that can be used. ECG model is a model by consider the signal in time domain and divides that signal following orderly progression of depolarization as shown in Fig. 1.

From Fig. 1 shows ECG signal model format as typical ECG signal. It can be seen that the normal ECG

\footnotetext{
+ Corresponding author. Tel.: + 66866230864.

E-mail address: kai_2521@hotmail.com.
} 
waveform consists of a P wave, a QRS complex and a T wave (U wave doesn't consider since it has lower amplitude and is hidden by $\mathrm{T}$ wave).

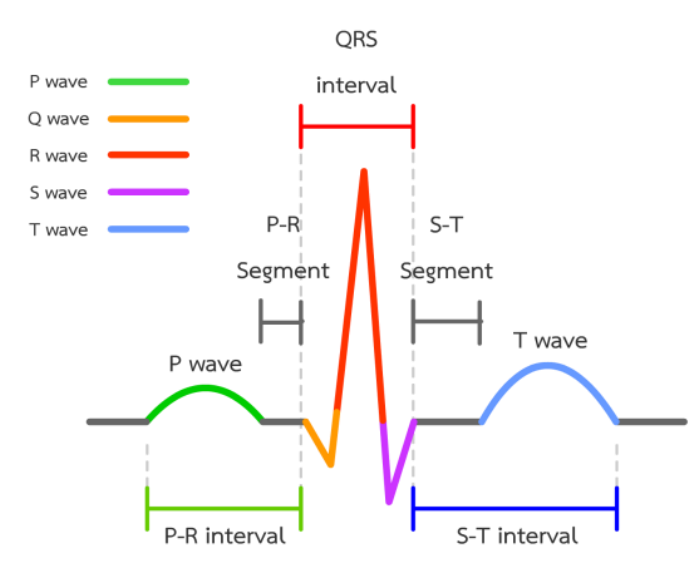

Fig. 1: ECG signal model format.

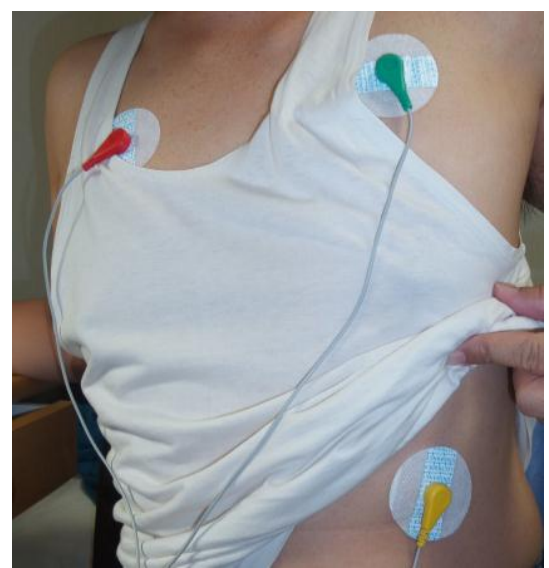

Fig. 2: ECG Leads II measurement.

\subsection{ECG signal measurement}

To obtain the heart signal in ECG format can measure electrical voltage from human body called "electrode". Electrode is a probe that can stick into human skin and can obtain voltage called "Leads II ECG" as in Fig. 2.

From Fig. 2 shown ECG Leads II measurement that is typical data of heart signal. It must use at least two probes to obtain signal because it has to measure differential electrical signal between two points on body and use other probes to reduce noise and emphasize some part of the signal.

\subsection{Analysis method}

Heart signal is a periodic signal meaning the signal was produced in full waveform periodically, but it is different from the normal waveform sine wave. Because there are many components in this signal produce for the different activity make this waveform very complicate. To analyse ECG waveform you have to develop a system to detect the identity of each waveform [5][6]. The proposed system is designed to detect the $\mathrm{R}$ peak of the signal. Because $\mathrm{R}$ peak will let us know about start and stop of heart beat waveform and we also can estimate the heart rate from interval of $\mathrm{R}$ peak. The proposed system based on real-time system has to process data finish immediately data was obtained and must finish process current waveform before next waveform come. In this paper, the proposed system can be used combine technique to detect each waveform. The proposed system is three techniques: (as shown in Fig. 3.).

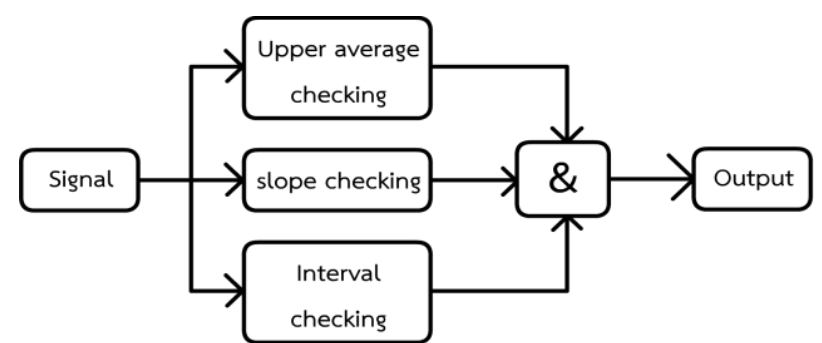

Fig. 3: Analysis method diagram.

- Upper average checking: the proposed system which knows the R peak of the signal is the highest amplitude of the signal always process average data of the signal using the complementary filter. And it must higher than 1.2 times of average signal so the complementary filter is designed for finding average values of signal at a time and use as one of feature to detect $\mathrm{R}$ peak.

- Slope checking: the processing every difference value of signal can calculate currently slope of signal by comparing current slope and latest slope. The peak of signal can be considered because if there is peak it is slope must less than 1 and the latest slope must more than 1.

- Interval checking: the human heart that has the heart rate more than 200 times per minute or period of $\mathrm{R}$ peak interval must more than $300 \mathrm{~ms}$ is measured. 


\section{System architecture}

\subsection{Component}

The proposed system contains electrode, pre-processing unit and processor. Electrode is a group of instrument that obtain heart signal from the human body as above. Pre-processing unit is a device that connects directly to electrode for obtaining heart signal. The heart signal is a low level signal (the signal range is $-2 \mathrm{mV}$ to $2 \mathrm{mV}$ ) so it has to be filtered and amplified for compatible with microcontroller and resist noise. The proposed system Heart Rate Monitor Front End IC name "AD8232" this IC designed to prepare biopotential small signals for ADC in the microcontroller to acquire that signal [7]. AD8232 contains a differential amplifier for comparing signals from electrode and transform into ECG format. Then there is a two-pole high pass filter for eliminating noise and extract the pure signal from the electrode. After that there is fast restores function amplifier for amplifying the signal to $3 \mathrm{~V}$ p-p for compatibility with typical microcontroller.

In processor unit, the proposed system is used the STM32F7 Discovery board as a microcontroller [8]. Discovery board contains stm32f746g microcontroller. This microcontroller is ARM 32-bit Cortex-M7 that can run at $200 \mathrm{MHz}$ and there is FPU (Floating-point unit) to calculate floating-point value for the faster DSP application. Moreover, microcontroller on Discovery board still has another necessary component for developing such as Display for show data programmer for flashing new firmware.

\subsection{Interfacing}

Electrode has been stacked directly to skin and their signal has connected to the AD8232 pre-processing unit. It produces high level enough signal for the microcontroller to acquiring via 12-bit ADC. Hence, the microcontroller can obtain a signal in the digital data format of ECG signal from heart muscle as shown in Fig 4.

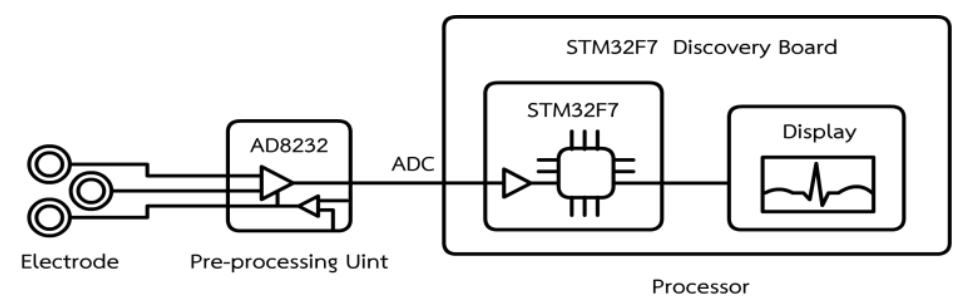

Fig. 4: Overall system.

\section{Experiment and Result}

The proposed system processes a Heart signal in real-time and show data (waveform and heart beat rate) via display as shown in Fig. 5. Because it detects $\mathrm{R}$ peak by above algorithm and uses a complementary filter to soften the heart rate value. Therefore, the proposed system can calculate it back into the heart beat rate and also align signal waveform to the center of display as seen on display [9]. The result of the proposed system can compare with other device such as wearable heart rate monitor as shown in Fig. 6.

From Fig. 6 shows the comparison between the results of the proposed system and the wearable device. It can be concluded that the both waveforms is similar. Table 1 shows the values of the proposed system and the values are obtained from wearable device for 15 times. It stated that the values are a very closely as shown in Fig. 7.

Table 1. Comparison Values between the Proposed System and Wearable Device

\begin{tabular}{|c|c|c|c|c|c|c|c|c|c|c|c|c|c|c|c|}
\hline & 1 & 2 & 3 & 4 & 5 & 6 & 7 & 8 & 9 & 10 & 11 & 12 & 13 & 14 & 15 \\
\hline Wearable & 72 & 78 & 75 & 73 & 84 & 73 & 75 & 74 & 77 & 83 & 77 & 74 & 75 & 77 & 79 \\
\hline our system & 72.6 & 77.3 & 77.3 & 77.2 & 83.9 & 74.4 & 78.5 & 75.4 & 78.8 & 82.8 & 80.9 & 76.4 & 77.0 & 78.4 & 82.4 \\
\hline
\end{tabular}

From Fig. 7 shows the graph comparison between the proposed system and wearable device. It is seen that some data are different because it measures the heart rate by calculate interval of $R$ peak of each wave. But this wearable device uses counting $\mathrm{R}$ peak in period that it makes value look difference in the same time. 


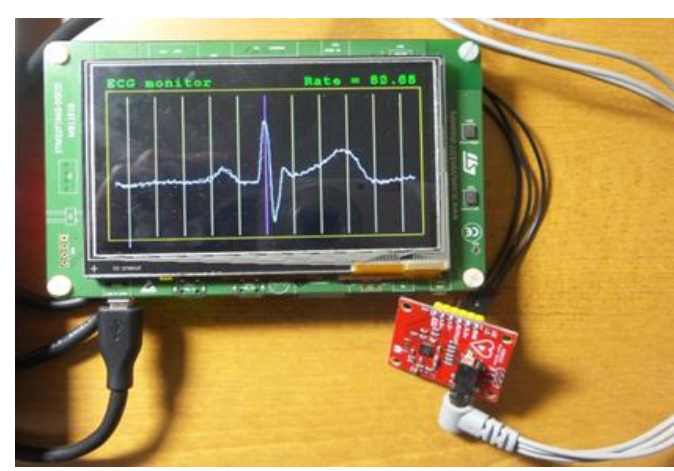

Fig. 5: Result waveform.

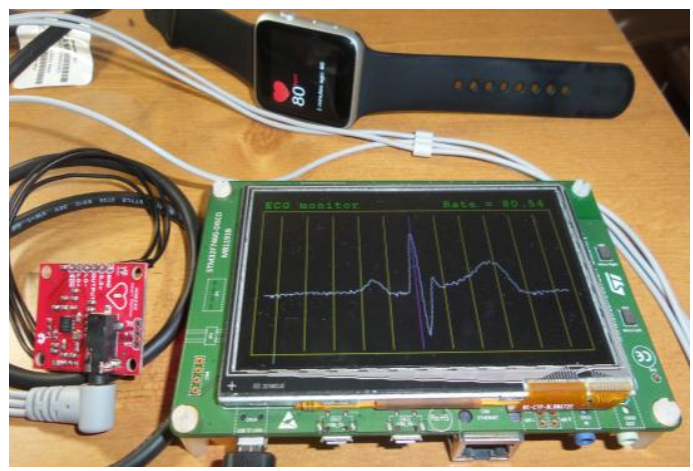

Fig. 6: Comparison with wearable device.

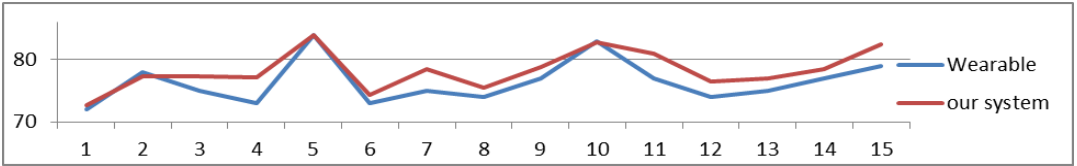

Fig. 7: Comparison graph.

\section{Conclusion}

In this paper, the system that can acquire an electrical heart signal from human body and can process to detect $\mathrm{R}$ peak to find heart beat rate has developed using stm32f7 microcontroller. The proposed system can be run on Li-ion battery with $2500 \mathrm{mAh}$ capacity during a day as same as a typical ECG monitoring. Moreover, it can't measure signal while person moving and can't be used in high level noise or high electromagnetic field area. Because heart signal is a low level signal; furthermore, wire and amplifier can be disturbed. In the future, the system will be developed by increase number of feature, develop pre-process unit include electrode to resist the noise batter, and also plan to test better by use real medical device ECG measurement as the measure heart rate .

\section{Acknowledgements}

This work was supported by KMITL-UEC Global Alliance Lab Project 2015. We would like to express my sincere thanks to Prof. Dr. Hisayuki AOYAMA. Furthermore, we also sincerely thank Associate Professor Dr. Kanok Janchitrapongvej for his teaching and advice.

\section{Reference}

[1] V.Chutchavong, K.Nualon, O. Sangaroon and K. Janchitrapongvej. A mathematical model for ECG waveform using rational Bézier curves and Bernstein polynomials.Electrical Proc. of ECTI-CON 2014, 11th International Conference. 2014

[2] Osman Özkaraca; Ali Hakan Işik; İnan Güler. Detection, real time processing and monitoring of ECG signal with a wearable system Proc.of Innovations in Intelligent Systems and Applications (INISTA), 2011 International Symposium.2014

[3] Raquel Gutiérrez-Rivas; Juan Jesús García; William P. Marnane; Álvaro Hernández.Novel Real-Time Low Complexity QRS Complex Detector Based on Adaptive Thresholding. IEEE Sensors Journal 2015

[4] Ministry of Public Health: http://www.moph.go.th/

[5] Dhanashri H Gawali; Vijay M. Wadhai. Implementation of ECG sensor for real time signal processing applications.Proc. of Advances in Electronics, Computers and Communications (ICAECC), 2014 International Conference on 2014

[6] Moumen Cherif; Malika-Djahida Kedir-Talha; Malika Tighidet. Acquisition and processing on DSP of a cardiac signal Proc.of Information and Communication Systems (ICICS), 2014 5th International Conference on 2014

[7] Analog devices :www.analog.com

[8] Stmicroelectronics: www.st.com

[9] Apple Watch. Online: https://www.apple.com/watch 